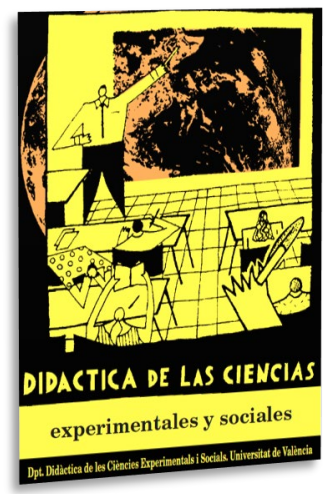

\title{
Valoración del profesorado en formación de educación infantil sobre su participación activa en itinerarios patrimoniales
}

\author{
Assessment of teachers in early childhood \\ education training on their active \\ participation in heritage itineraries
}

DOI: $10.7203 / D C E S .39 .17020$

\author{
José Antonio López Fernández \\ Universidad de Córdoba, jalopez@uco.es \\ ORCID iD: https://orcid.org/0000-0002-1779-5976 \\ Nuria Fernández Terán \\ Universidad de Córdoba, nuriaft94@gmail.com
}

\begin{abstract}
RESUMEN: La investigación de lo que ocurre en las aulas de formación del profesorado de educación infantil cuando se trabaja el patrimonio cultural es todavía poco significativa con respecto a primaria y secundaria. En este artículo se presenta un análisis sobre las valoraciones de una propuesta concreta, con alumnado en formación inicial de educación infantil, participante en un itinerario didáctico de carácter patrimonial, donde éste interactúa en todos los procesos didácticos. El análisis se ha realizado en un grupo concreto, con 52 estudiantes de la Universidad de Córdoba. El objetivo es evaluar las aportaciones conceptuales y competenciales de esta práctica docente para la formación del futuro profesorado. Se trata de un estudio exploratorio y descriptivo, con dos herramientas para la toma de datos: un cuestionario semiestructurado y preguntas cerradas; y un estudio focal. Los resultados son bastante positivos, si bien es necesario seguir considerando una educación patrimonial globalizada e interdisciplinar, dejando de lado metodologías de contemplación y exaltación de lo monumental.
\end{abstract}

PAlabras Clave: educación patrimonial, formación del profesorado, itinerario didáctico patrimonial, educación infantil, Universidad de Córdoba

ABSTRACT: Research on what happens in early childhood teacher training classrooms when working on cultural heritage is still of little significance with respect to primary and secondary education. This work presents an analysis of the evaluations of a specific proposal, with students in initial training in early childhood education, participating in a heritage-based didactic itinerary, with trainees interacting in all the didactic processes. The research has been carried out in a specific group, with 52 students from the University of Cordoba. The objective is to evaluate the conceptual and competence contributions of this teaching practice to the training of future teachers. It is an exploratory and descriptive study, with two tools for data collection: a semi-structured questionnaire and closed questions; and a focal study. The results are quite positive, although it is necessary to continue considering a globalized and interdisciplinary heritage education, leaving aside methodologies of contemplation and exaltation of monumental heritage.

KEYWORDS: heritage education, teacher training, heritage didactic itinerary, childhood education, University of Cordoba

Fecha de recepción: abril de 2020

Fecha de aceptación: mayo de 2020

Este trabajo se inscribe en el marco del Proyecto de Investigación PATTERN. "Patrimonio Arqueológico, Nuevas Tecnologías, Turismo, Educación y Rentabilización Social: un nexo necesario para la ciudad histórica”, financiado por la Dirección General de Investigación Científica y Técnica y Subdirección General de Proyectos de Investigación. Ministerio de Economía y Competitividad. Gobierno de España, en su convocatoria de 2015 (Ref.: HAR2015-68059-C2-1-R; Subprograma Retos de la Sociedad). 


\section{INTRODUCCIÓN}

En los últimos años se viene tratando la educación patrimonial desde la didáctica de las Ciencias Sociales en la formación del profesorado de educación infantil y primaria, a través de diferentes metodologías didácticas, de carácter activo, y aprovechando el contacto directo con los recursos naturales y culturales (Medina y López, 2018; Medina, Arrebola, Mora y López, 2016; Coma, 2012). En ciudades patrimoniales, como Córdoba, el patrimonio se convierte en un recurso per se en la formación del profesorado. Entendemos la educación en torno al patrimonio como un campo disciplinar (Fontal, 2007), que puede funcionar de modo interdisciplinar (Cuenca, 2003; Estepa, Wamba y Jiménez, 2005) para conocer el medio desde edades tempranas e introducir metaconceptos como el tiempo y el espacio.

Tradicionalmente se ha considerado que el alumnado de educación infantil no está capacitado para trabajar estos conceptos (Piaget, 1954; Inhelder, Bovet, Sinclair y Smock, 1966). Sin embargo, el uso de diferentes metodologías a través del patrimonio ofrece otros datos (Eisner, 1994; Egan, 1991; Tonda, 2001; Trepat y Comes, 2002; Moreno y Vera, 2016; Arias y Casanova, 2018; Arias, Casanova, Egea, García y Morales, 2016; Casanova, Arias y Egea, 2018; González-Monfort, 2006 y 2019; Teixeira, 2006; Casanova y Casanova, 2014; Moreno y Gallego, 2010). De este modo, es necesario considerar una formación del profesorado que capacite al estudiantado para integrar la educación patrimonial en sus competencias profesionales, alejada de metodologías tradicionales y transmisivas.

Situados en la perspectiva de una educación patrimonial como eje transversal (Fuertes, 2016; Cuenca, 2003), planteamos una investigación sobre las valoraciones e influencia que presenta un itinerario didáctico de carácter activo con profesorado en formación de educación infantil (Delgado y Alario, 1994; Insa, 2002; López y Segura, 2013; Santolaria, 2014; Vilarrasa, 2003a y 2003b; Piotrowska, Cichoń, Abramowicz y Sypniewski, 2019; Mateo, 2019; Ferreras-Listán, MorenoFernández y Puig, 2019); un nivel en el que existen numerosas propuestas pero escasean estudios y resultados sobre la influencia de prácticas docentes concretas.

Las sensaciones de la secuencia didáctica implementada son positivas a lo largo de estos años (Figura 1). No obstante, se han querido analizar las concepciones que ofrecen las protagonistas que participan durante un cuatrimestre en una asignatura de carácter optativo, Patrimonio históricoartístico y escuela. Se pretende recabar información sobre la planificación de esta actividad, la valoración metodológica, la predisposición de los espacios y tiempos destinados a cada evento, así como su operatividad de cara a la educación infantil. Para ello, hemos utilizado un cuestionario de carácter cuantitativo, por medio de la confección de una escala de satisfacción, y un estudio focal de índole cualitativo.

FIGURA 1. Estudiantes en formación del profesorado de educación infantil interactuando en el itinerario

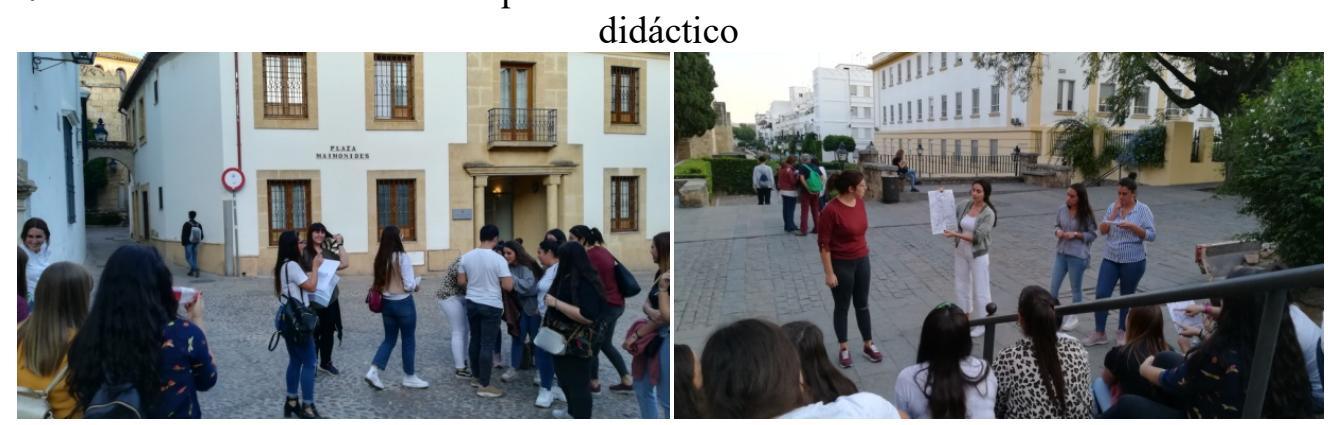

Fuente: Elaboración propia

El punto inicial de la investigación se posiciona en la representación social que el patrimonio tiene en la actualidad en la formación del profesorado (Cuenca, 2003; Estepa, Cuenca y Ávila, 2006; Franco y Huerta, 2011; Molina y Ortuño, 2017; Miralles, Gómez y Rodríguez, 2017; López, 
2020; Castro y López-Facal, 2019); una concepción que no va más allá de una visión tradicional y monumental (Fontal e Ibáñez, 2014; Cuenca, 2003) al inicio de sus estudios universitarios.

Al contrario, la propuesta a analizar parte de la finalidad de una educación patrimonial para formar a futuros y futuras docentes en la resolución de los problemas sociales y la formación crítica de la ciudadanía (Cuenca, 2014). Por otro lado, también se intenta obtener información relevante sobre las propias prácticas docentes relacionadas con contextos de aprendizaje diferentes al aula (Pagès y Santisteban, 2014).

\section{MARCO TEÓRICO}

\subsection{La educación patrimonial desde la didáctica de las Ciencias Sociales}

La concepción actual de la educación patrimonial pretende dar a entender los hechos espaciales, sociales e históricos como causantes de la realidad presente (Ballesteros, Fernández, Molina y Moreno, 2003; Hernández, 2003; Prats, 2003; Estepa, 2009; Miralles, Molina y Ortuño, 2011; Ibáñez, Fontal y Cuenca, 2015; Cambil y Tudela, 2017); reflejo de un legado simbólico e identitario que se debe trasladar a las próximas generaciones.

El patrimonio, no obstante, constituye un concepto diverso, que ha evolucionado a lo largo de los siglos y que varía en función del campo o área de conocimiento que se acerca a su tratamiento (Cambil y Fernández; 2017). En las Ciencias Sociales, la geografía y la historia, según GonzálezMonfort (2007; 2019), el patrimonio constituye un constructo social e integrador donde poder aprender a valorar y comprender el funcionamiento de un colectivo a lo largo del tiempo, aportando un caudal de información para el conocimiento histórico y social.

Siguiendo a Fontal (2003), Cambil y Fernández (2017) destacan que la educación patrimonial requiere que se lleve a cabo en y desde el patrimonio, donde es esencial el papel de la escuela ya que es en ella donde debe iniciarse "el proceso de patrimonialización, entendido no sólo como el análisis y el estudio de los elementos patrimoniales, sino como la relación de pertenencia, propiedad e identidad que se genera entre determinados bienes y personas" (p. 33). Sin embargo, en la escuela, al patrimonio se le ha otorgado habitualmente una finalidad meramente complementaria y fragmentada; situación mostrada tanto en los libros de textos -donde se evidencia un escaso tratamiento- como en los diferentes currículos (Cuenca y Estepa, 2003; González-Monfort, 2011; Hernández y Guillén, 2017; Pinto y Molina, 2015; Fontal, Ibáñez, Martínez y Rivero, 2017). Una visión monumental, estética y descriptiva que sigue perseverando entre el profesorado en formación y en activo (Cuenca, 2003; Molina y Muñoz, 2016).

Al contrario, las investigaciones y propuestas recientes plantean el patrimonio desde un punto de vista multidisciplinar, holístico y vivencial, a través del cual es posible vertebrar todo un proyecto curricular (Cuenca y Domínguez, 2000; Candreva y Susacasa, 2003; Prats, 2001; Cuenca, 2014; Cuenca, Estepa y Martin, 2017; Morón y Morón, 2017; Arias y Casanova, 2018). Los últimos avances señalan que el tratamiento didáctico constructivista en torno al patrimonio puede ayudar a desarrollar numerosos conceptos temporales, como la duración, el cambio o la continuidad (Pagès y Santisteban, 2010), fomentar la conciencia ciudadana (Cuenca, 2014) además de mostrar el patrimonio como fuente histórica de carácter primario (Miralles y Rivero, 2012).

En este sentido, los planteamientos holísticos y cooperativos que se vienen desarrollando en las aulas de infantil, permiten ayudar al alumnado a descubrir el entorno (Feliu, Jiménez y Arqué, 2014) a través de diferentes metodologías, como los proyectos de trabajo (Casanova y Casanova, 2014; Casanova, Arias y Egea, 2018; Calderón, 2018), los itinerarios (Vilarrasa, 2003a y 2003b; Santolaria, 2014; Coma, 2012), o por medio del uso de las tecnologías de la información y la comunicación. En este proceso, resulta elemental una adecuada formación del profesorado para capacitar a maestras y maestros en el uso y aplicación de diferentes metodologías e introducir así la educación patrimonial (Pagès, 2000). Una formación que es la primera inquietud de los docentes, 
además de contar con recursos, programas y proyectos de innovación educativa, según se desprende de los resultados del Plan Nacional de Educación y Patrimonio (PNEP, 2015).

\subsection{La investigación sobre contextos de aprendizaje relacionados con el patrimonio cultural en la formación del profesorado}

La investigación relacionada con la educación patrimonial se ha desarrollado en los últimos años en torno al análisis de materiales curriculares, las concepciones del profesorado en formación, o en relación al desarrollo de capacidades y competencias, entre ellas el pensamiento histórico (Domínguez y López, 2017; Chaparro, Martínez, Robles y Cespedosa, 2019; Santacana y Martínez, 2013). Otra línea muy potente sobre la educación patrimonial es la que concierne al aprovechamiento didáctico de museos o la recreación de espacios musealizados en el aula (Cuenca, Martín y Schugurensky, 2017; Santacana, 2006; Fernández, 2003; Estepa, Ávila y Ruíz, 2007; Santacana, Martínez, Llonch y López, 2016; Gómez, 2014; Escribano, 2015; Cuenca y Martín, 2014; Ávila y Duarte, 2014). Sin embargo, la investigación didáctica de lo que ocurre en la formación docente cuando se aplican diversas metodologías son poco representativas, sobre todo con alumnado en formación inicial de educación infantil (Miralles, Molina y Ortuño, 2011).

Lleida (2010) estudia el uso del patrimonio arquitectónico con profesorado en activo de Ciencias Sociales, Geografía e Historia, de educación secundaria, donde observa el predominio de un modelo didáctico tradicional sin que el patrimonio constituya una fuente histórica. Resultados que ya reflejó González-Monfort (2008) al destacar que el patrimonio se presenta como algo puntual en las aulas de secundaria en Cataluña, con prácticas docentes tradicionales y actividades lúdicas de escaso valor educativo.

Miralles, Gómez y Rodríguez (2017) analizan las valoraciones de docentes en formación de educación secundaria de España e Inglaterra sobre diferentes estrategias metodológicas. Al respecto, las salidas a centros de interés histórico y el trabajo de campo reciben una elevada valoración, más acentuada en el caso español. Los investigadores indican que el profesorado en formación de esta etapa educativa considera el patrimonio muy positivamente como un recurso didáctico o estrategia metodológica para la enseñanza de la historia. Potencial didáctico también evidenciado en la formación del profesorado en Portugal (Alves y Pinto, 2019). Duarte y Ávila (2015) proponen en este sentido un modelo didáctico basado en la investigación para trabajar el patrimonio. Luna, Vicent, Reyes y Quiñonez (2019), por su parte, estudian la presencia del patrimonio en la legislación educativa mexicana y su incidencia en los planes de estudio de formación del profesorado de educación primaria.

Respecto a educación infantil, se han publicado varias experiencias y propuestas pero, como venimos señalando, son pocos los análisis sobre el impacto o la mejora en el proceso de enseñanza y aprendizaje. A destacar los trabajos recientes de Ferreras-Listán, Moreno-Fernández y Puig (2019), y de Bonilla y Lucena (2019), que evalúan varias prácticas con alumnado en formación de educación infantil en relación con el patrimonio tomando la información de los propios estudiantes $\mathrm{y}$ donde destacan, entre otras cuestiones, que las salidas escolares y los itinerarios supusieron un punto de inflexión en el desarrollo de sus competencias docentes, provocando aprendizajes significativos y un caudal de conocimientos trasladable a las aulas.

\section{Metodología}

\subsection{Objetivo e hipótesis}

El objetivo principal de la investigación reside en analizar la percepción del profesorado en formación de educación infantil sobre el tratamiento del patrimonio cultural, a través de itinerarios 
didácticos de carácter activo y patrimonial en el que el alumnado tiene un carácter participativo en todos los procesos de desarrollo (antes, durante y después).

La hipótesis de trabajo se concreta en una práctica aceptada positivamente por el alumnado. Aunque requiere un proceso amplio de preparación y una participación colaborativa, la opinión del alumnado induce que la propuesta permite una mejor adquisición de los contenidos, así como la asimilación de conceptos y procesos didácticos cuando se trabaja fuera del aula.

\subsection{Diseño de la investigación}

En primer lugar, se planteó un análisis descriptivo y cuantitativo, con la toma de datos al término del curso (final del primer cuatrimestre del curso 2019-2020) a través de un cuestionario semiestructurado y cerrado. Para complementar la toma de datos, se procedió a realizar un estudio focal. De las 56 personas que optaron por la asignatura, 52 han realizado el cuestionario $(\mathrm{n}=52)$, mientras que 4 fueron seleccionadas para desarrollar el estudio focal, procediéndose posteriormente a la triangulación de los datos. Las personas participantes nos permiten asegurar un promedio aceptable de los resultados, con un nivel de confianza del $99 \%$ y un margen de error del 5\%.

El escenario a investigar es el planteamiento de un itinerario didáctico que se enfoca, no solamente para dar a conocer el patrimonio cultural de Córdoba, sino como recurso participativo y formativo del estudiantado. El profesorado encargado presenta las particularidades conceptuales de un itinerario como recurso didáctico, y diseña un recorrido con paradas en puntos emblemáticos de la ciudad, a las que se le añaden distintas temáticas a trabajar (Tabla 1).

Tabla 1. Diseño y desarrollo del itinerario didáctico que supone el contexto de la investigación

\begin{tabular}{ccc}
\multicolumn{4}{c}{ Tabla 1. Diseño y desarrollo del itinerario didáctico que supone el contexto de la investigación } \\
\hline Espacio: Aula & \multicolumn{1}{c}{ Diseño del itinerario didáctico } & Tiempo: 2 semanas \\
\hline & Docente: Diseño y coordinación & \\
& - Tratamiento de los conceptos que integran el patrimonio, su potencial didáctico y contribución \\
en la formación de la ciudadanía. & \\
- Utilidades relacionadas con el acercamiento al alumnado de infantil. & \\
- Organización del trabajo fuera del aula. Distribución de paradas en relación al número de & subgrupos en el que se divide el grupo clase (7 grupos pequeños)
\end{tabular}

\begin{tabular}{|c|c|c|}
\hline \multicolumn{3}{|r|}{ Desarrollo del itinerario didáctico } \\
\hline & cio: Casco histórico de Córo & Docente: Orientación \\
\hline \multicolumn{3}{|r|}{ Tiempo: una jornada } \\
\hline \multicolumn{2}{|c|}{ Punto de parada } & Temática \\
\hline & Puente Romano & $\begin{array}{l}\text { El puente, vías de comunicación romanas e importancia en torno a la } \\
\text { localización del río }\end{array}$ \\
\hline 2 & Sotos Albolafia & Uso tradicional del agua. Molinos hidráulicos. Biodiversidad del río \\
\hline & Barrio de San Basilio & $\begin{array}{l}\text { Importancia histórica y social de los patios de Córdoba. Los patios como } \\
\text { lugar de convivencia }\end{array}$ \\
\hline 4 & Alcázar Reyes Cristianos & El edificio y sus jardines. Elemento turístico en la actualidad \\
\hline 5 & Plaza Maimónides & Los judíos en Córdoba. Crisis religiosas actuales \\
\hline 6 & Puerta de Almodóvar & Las murallas de la ciudad. De elemento defensivo a recurso patrimonial \\
\hline & $\begin{array}{l}\text { Cisternas romanas de } \\
\text { Conde Vallellano }\end{array}$ & Abastecimientos tradicionales de agua procedentes de la sierra \\
\hline
\end{tabular}

Fuente: Elaboración propia

Se trata de un recorrido multidisciplinar y diacrónico, ya que los puntos de atención alternan diferentes lugares históricos, de diversas épocas, pero que llegan hasta la actualidad con la consideración de patrimonio con variables tangibles e intangibles, aunque con distintos usos y estados de conservación. A partir de este proceso, el alumnado, por grupos, es el encargado de 
abordar el resto de fases metodológicas. Antes, debe indagar sobre dicha temática, que engloba la historia y evolución de ese lugar concreto, coordinar la búsqueda de información y sistematizarla. En la fase de implementación, tiene que presentarla in situ al resto de compañeras y compañeros con la selección de recursos que estimen oportunos, además de proponer actividades a desarrollar en el propio recorrido, lo que supone la búsqueda y diseño de materiales. Después, por medio de la elaboración de una ficha que incluye una reflexión de todo el proceso, y que supone una retroalimentación grupal final en clase.

\subsection{Instrumentos de recogida de información}

Los instrumentos que se han utilizado han sido un cuestionario y un estudio focal. En el primer caso, se ha optado por disponer preguntas cerradas con escala Likert, con opciones desplegadas entre 1 y 5, siendo 1 totalmente en desacuerdo, 2 en desacuerdo, 3 indiferente, 4 de acuerdo y 5 totalmente de acuerdo. Para su validez, se realizó un primer cuestionario del que se eliminaron algunas consideraciones por su reducida operatividad en la investigación. En la versión final, los items del 1 al 3 hacen referencia a la mejora que puede provocar esta metodología en la formación docente; del 4 al 6 se pregunta sobre el tiempo destinado a la preparación de la actividad, así como la idoneidad didáctica de los espacios utilizados en los puntos de parada; 7 y 8 abordan los conocimientos factuales provocados por la actividad; y el 9 se relaciona con la aportación de la actividad para la adquisición de competencias espaciales y temporales por parte del alumnado de educación infantil. Respecto al análisis de fiabilidad, obtenido a partir del alfa de Cronbach y realizado con el programa informático IBM SPSS Statistic 25, este otorga un valor de ,864; lo que indica una buena consistencia a partir del conjunto de ítems desarrollados (Tabla 2).

TABLA 2. Resultados de fiabilidad, obtenido con el programa informático SPSS Statistic 25

\begin{tabular}{ccc}
\hline Alfa de Cronbach & $\begin{array}{c}\text { Alfa de Cronbach basada en } \\
\text { elementos estandarizados }\end{array}$ & N de elementos \\
\hline .864 & .886 & 9 \\
\hline
\end{tabular}

El focus group, por su parte, se realizó con 4 alumnas seleccionadas al azar, para que valorasen la actividad. Este procedimiento se llevó a cabo una vez analizados los datos de los cuestionarios. Las preguntas del mismo, no obstante, se centraron en los cuatro apartados o dimensiones del cuestionario, para contrastar la información aportada por estos, buscando respuestas a cuestiones concretas como la atención durante los recorridos o la dificultad en la búsqueda de información.

\section{RESULTADOS}

Los resultados son positivos según la media y la desviación estándar determinadas para cada dimensión (Tabla 3), aunque con algunas diferencias que pasamos a comentar. La pregunta 1 solicita una valoración sobre la mejora que produce la metodología aplicada en la actividad docente al trabajar el patrimonio cultural. La pregunta 2, si tendrá en cuenta esta metodología en su futuro como docente y la cuestión $n^{0} 3$ evalúa el grado de satisfacción a la hora de tener que buscar información y presentarla al resto de compañeras y compañeros (Tabla 4). Los valores medios de estas tres cuestiones se sitúan entre 4,71 y 4,90 sobre 5. En la primera cuestión, el $98 \%$ está de acuerdo y, sobre todo, totalmente de acuerdo en la metodología utilizada. Además de romper la rutina del aula, la mayoría entiende que mejora su formación docente; una consideración que redunda en los resultados de la pregunta $\mathrm{n}^{\circ} 2$, ya que el $90 \%$ está de acuerdo en tenerla en cuenta para su futura implementación en las aulas de educación infantil. En el estudio focal, por su parte, se acentúa el interés de esta metodología, así como una mayor motivación provocada por el cambio 
de rutinas del aula y el hacer vivenciales muchos aspectos del patrimonio y la historia local (PNEP, 2015).

TABLA 3. Datos estadísticos sobre la media y desviación estándar

\begin{tabular}{|c|c|c|c|}
\hline & Item & Media & $\begin{array}{c}\text { Desviación } \\
\text { estándar }\end{array}$ \\
\hline \multirow{3}{*}{ Mejora de la formación docente } & 1 & 4.83 & 0.43 \\
\hline & 2 & 4.9 & 0.3 \\
\hline & 3 & 4.71 & 0.54 \\
\hline \multirow{3}{*}{ Tiempos y espacios utilizados } & 4 & 4.46 & 0.67 \\
\hline & 5 & 4.44 & 0.64 \\
\hline & 6 & 4.77 & 0.43 \\
\hline \multirow{2}{*}{ Conocimientos factuales } & 7 & 4.83 & 0.43 \\
\hline & 8 & 4.87 & 0.34 \\
\hline Utilidad del recurso y la metodología para educación infantil & 9 & 4.87 & 0.34 \\
\hline
\end{tabular}

TABLA 4. Valoraciones sobre la mejora de la formación que supone la actividad de cara a formación docente del alumnado de educación infantil

\begin{tabular}{|c|c|c|c|c|c|}
\hline CUESTIÓN & 5 & 4 & 3 & 2 & 1 \\
\hline $\begin{array}{l}\text { 1. Este tipo de prácticas mejoran la formación de cara a mi } \\
\text { futura actividad docente }\end{array}$ & 84.62 & 13.46 & 1.92 & & \\
\hline $\begin{array}{l}\text { 2. Tendré en cuenta la metodología de trabajar fuera del } \\
\text { aula en mi futura labor docente }\end{array}$ & 90.38 & 9.62 & & & \\
\hline $\begin{array}{l}\text { 3. Tener que buscar información y presentarla al resto de } \\
\text { compañeros ha mejorado mi conocimiento sobre el } \\
\text { patrimonio de Córdoba }\end{array}$ & 75.00 & 21.15 & 3.85 & & \\
\hline
\end{tabular}

Fuente: Elaboración propia

Respecto a la tercera cuestión, el alumnado está totalmente de acuerdo (75\%) y de acuerdo (21\%) en que la metodología propuesta supone una mejora de su conocimiento sobre aspectos históricos. A pesar de que la actividad se desarrolla en la propia ciudad en la que residen, muchos de los lugares estudiados les son desconocidos, así como su evolución histórica. El focus group evidencia que su participación en la búsqueda y exposición de los contenidos, a diferencia de ser meros expectadores, mejora sus conocimientos patrimoniales y didácticos.

En cuanto a los items del 4 al 6 (Tabla 5), el primero solicita una valoración sobre la preparación del itinerario. La cuestión 5, por su parte, cuestiona el tiempo del itinerario, una jornada de tarde con una duración de 3 horas y 30 minutos, con un total de 7 paradas de 15-20 minutos cada una, donde por grupos debe exponerse la temática y desarrollar una actividad. Por último, la pregunta 6 indaga sobre la idoneidad de los espacios utilizados para llevar a cabo la tarea.

TABLA 5. Valoración de las cuestiones relacionadas con el tiempo y el espacio utilizados para el desarrollo de la actividad

\begin{tabular}{lccccc}
\hline CUESTIÓN & $\mathbf{5}$ & $\mathbf{4}$ & $\mathbf{3}$ & $\mathbf{2}$ & $\mathbf{1}$ \\
\hline $\begin{array}{l}\text { 4. El tiempo destinado a la preparación de las } \\
\text { actividades fuera del aula han sido adecuados }\end{array}$ & 55.77 & 34.62 & 9.62 & & \\
\hline $\begin{array}{l}\text { 5. La actividad que se realizó fuera del aula ha } \\
\text { sido adecuada para la formación del profesorado }\end{array}$ & 51.92 & 40.38 & 7.69 & & \\
\hline $\begin{array}{l}\text { 6. Los espacios sobre los que se han realizado las } \\
\text { prácticas son adecuados }\end{array}$ & 76.92 & 23.08 & & & \\
\hline
\end{tabular}

Fuente: Elaboración propia 
En las cuestiones que indagan sobre el tiempo destinado a la preparación de la actividad, la duración y los espacios utilizados, los resultados siguen siendo bastante positivos, aunque con una valoración media algo inferior, entre 4,44 y 4,77, sobre 5 . Más del $50 \%$ está totalmente de acuerdo con el tiempo asignado, junto al 34\% que está de acuerdo. Mientras, un 10\% se siente indiferente. Expuesta esta cuestión en el estudio focal, las participantes manifestaron la idoneidad del tiempo acotado a la preparación de la actividad. Las dificultades iniciales que plantea tener que buscar y seleccionar la información de forma autónoma, aunque es un proceso guiado, se convierten posteriormente en un valor positivo de la actividad, ya que permite desarrollar destrezas a la hora de elegir las fuentes de información y poder contrastarlas. El análisis focal evidencia, no obstante, que una parte del alumnado se sigue encontrando más cómodo ante actividades más pautadas, que no requieren el desarrollo de este tipo de destrezas y que, a pesar de las críticas, inciden en un enfoque tradicional de simple memorización y exposición.

La pregunta 5, que versa sobre el tiempo destinado a la sesión de trabajo en el itinerario y la propia actuación de cada grupo, muestra buenos resultados por parte del alumnado, aunque un $8 \%$ se muestra ni de acuerdo ni en desacuerdo con esta cuestión. Si bien la actividad es larga, la predisposición de los grupos, su metodología de exposición y las actividades desarrolladas son valoradas positivamente en el estudio focal. Sin embargo, las propuestas y exposiciones tradicionales de algún grupo acentúan la pérdida de atención y la percepción de que el itinerario se alarga. A ello se suma la desmotivación de los grupos que exponen al principio de la actividad y que se relajan posteriormente.

La pregunta 6 presenta la valoración de los lugares por donde discurre el itinerario. Los resultados señalan la visión positiva del alumnado, espacios de gran valor patrimonial y turístico. Además, como se señaló anteriormente, a pesar de ser lugares de interés, muchos aspectos les resultan hasta entonces desconocidos e invisibilizados. Así, la propuesta de indagación y exposición al resto del alumnado permite un mayor conocimiento histórico $\mathrm{y}$, en cierto modo, un cambio valorativo e interpretativo del entorno.

Los siguientes resultados se relacionan con las preguntas 7 y 8 (Tabla 6).

TABLA 6. Valoraciones sobre el aprendizaje factual que produce la metodología activa del itinerario didáctico en la formación del profesorado

\begin{tabular}{lccccc}
\hline CUESTIÓN & $\mathbf{5}$ & $\mathbf{4}$ & $\mathbf{3}$ & $\mathbf{2}$ & $\mathbf{1}$ \\
\hline $\begin{array}{l}\text { 7. He podido aprender características de } \\
\text { espacios que desconocía }\end{array}$ & 84.62 & 13.46 & 1.92 & & \\
\hline $\begin{array}{l}\text { 8. Tomar contacto directo con los elementos } \\
\text { patrimoniales me ha permitido mejorar mi } \\
\text { conocimiento sobre la historia y el patrimonio }\end{array}$ & 86.54 & 13.46 & & & \\
\hline
\end{tabular}

Fuente: Elaboración propia

En ellas, se cuestiona sobre la incidencia de la metodología en el aprendizaje factual de aspectos y características patrimoniales y el grado de valoración que tiene el contacto directo en este proceso.

El profesorado en formación está de acuerdo y totalmente de acuerdo en la metodología utilizada para aprender las características patrimoniales de su entorno cercano $(4,83$ y 4,87 respectivamente). Trabajar de forma colaborativa por medio de la indagación y preparar una exposición al resto del alumnado, aunque al principio se muestra como un reto, favorece el conocimiento del entorno cercano, de hechos y procesos históricos que dan sentido a la situación actual de la trama urbana. Además, como se anotó en el focus group, las actividades fuera del aula son muy reducidas en la formación del profesorado, cuando el discurso actual es preparar para la inserción en la vida real de una forma participativa y crítica, así como conocer y vivenciar el entorno para su protección ambiental y patrimonial. 
Por último, se interroga sobre las posibilidades de esta metodología para aplicarla en educación infantil con el objetivo de incluir nociones espaciales y temporales (Tabla 7) en su futuro alumnado.

TABLA 7. Valoraciones sobre la aplicabilidad del itinerario didáctico en la educación infantil

\begin{tabular}{lccccc}
\hline CUESTIÓN & $\mathbf{5}$ & $\mathbf{4}$ & $\mathbf{3}$ & $\mathbf{2}$ & $\mathbf{1}$ \\
\hline $\begin{array}{l}\text { 9. Con esta metodología, el alumnado de } \\
\text { educación infantil podrá adquirir competencias } \\
\text { espaciales y temporales }\end{array}$ & 86.54 & 13.46 & & & \\
\hline
\end{tabular}

Fuente: Elaboración propia

La valoración del profesorado en formación es positiva respecto a la idoneidad de utilizar el contacto directo con el entorno por medio de itinerarios didácticos para el desarrollo de competencias espaciales y temporales en educación infantil. Sin embargo, la trasposición didáctica de esta metodología llevada a las aulas con niñas y niños de 4 o 5 años debe adaptarse al estado madurativo de este alumnado, una cuestión que se puso de manifiesto en el análisis focal. En este grupo de trabajo consideran que la propuesta es interesante, que puede resultar bastante significativa, que el alumnado de educación infantil puede y está capacitado para trabajar fuera del aula (Pérez y Morón, 2016), pero que las actividades planteadas deben estar guiadas por el profesorado responsable, no solo en el desarrollo de la actividad (durante), sino también ayudando en la búsqueda de información, que debe ser mucho más reducida y sintética. Al respeto, también se ha sugerido la participitación de escolares en estas prácticas, como propuestas de mejora, y examinar en qué sentido se adapta la metodología y cómo se produce la adquisición de aprendizajes.

\section{DISCUSIÓN Y CONCLUSIONES}

Los avances en la didáctica de las Ciencias Sociales en general y del patrimonio en particular están aportando interesantes aportaciones en educación infantil, desde planteamientos sociohistóricos (Cuenca y Domínguez, 2000). Parece ser, pues, que los problemas de aprendizaje no residen tanto en la edad del alumnado, que ha de tenerse en cuenta obviamente, pero donde hay que revisar los contenidos, la metodología y los recursos didácticos utilizados (Trepat y Comes, 2000; Santisteban, 2000; Santisteban y Pagès, 2006). Las experiencias didácticas innovadoras en este sentido muestran avances, donde el alumnado es capaz de trabajar conceptos sociales, temporales y espaciales por medio de diversas metodologías como el agrupamiento por rincones, narraciones o el uso de objetos, entre otros (Pérez, Baeza y Miralles, 2008; De la Calle, 2011; Moreno y Vera, 2016; Arias y Casanova, 2018; Arias, Casanova, Egea, García y Morales, 2016; Casanova, Arias y Egea, 2018).

Al hilo de estas aportaciones, es necesaria una formación inicial del profesorado donde este adquiera competencias para abordar los procesos de enseñanza y aprendizaje con su futuro alumnado de educación infantil; competencias como capacitarle para reflexionar sobre lo que ocurre en el aula, a cuestionar los propósitos curriculares y replantear continuamente su concepción de la enseñanza y sus prácticas docentes (Tonda, 2001; Santisteban y Pagès, 2011).

Como hemos señalado, las teorías del desarrollo psicoevolutivo siguen presentes en la formación de las y los futuros docentes, algo que también se ha puesto de manifiesto a través de la investigación de su representación social sobre la enseñanza de la historia y las Ciencias Sociales en educación infantil (Hernández y Pagès, 2016). En nuestro trabajo, hemos querido evaluar si nuestra experiencia didáctica con el profesorado en formación de educación infantil favorece el conocimiento del entorno a través de la educación patrimonial y abandonar posturas tradicionales de observación y contemplación con respecto al patrimonio cultural. Se trata de una línea de 
investigación escasamente desarrollada, con mayor evaluación en otros niveles de la educación formal (Miralles, Molina y Ortuño, 2011).

Los datos de la percepción sobre nuestra propuesta metodológica para trabajar el patrimonio cultural, como parte de la asignatura Patrimonio histórico-artístico y escuela, son bastante positivos. El alumnado muestra su satisfacción de gran parte del proceso, que tiene como eje estructurante un itinerario didáctico en el que el estudiantado es partícipe en todas las fases del proceso (antes, durante y después) en torno a una educación patrimonial que permite conocer e interactuar con el entorno de forma activa.

Según su propia opinión, la propuesta didáctica mejora su formación docente respecto al conocimiento y valores que presentan los entornos patrimoniales de su espacio cercano; que este proceso les resulta motivador, a diferencia de las clases magistrales y de las prácticas tradicionales desarrolladas en el aula; y que se trata de un recurso interesante y aplicable a la etapa de educación infantil. Un proceso didáctico que mejora sus competencias profesionales (Ávila y Duarte, 2015).

Los datos del estudio focal han permitido asegurar y contrastar la información recogida en el cuestionario cuantitativo. De este modo, el uso de estos dos instrumentos permiten afirmar la valoración positiva de las participantes en el diseño y desarrollo del itinerario. Con ello, intentamos evitar una recogida de datos sesgada con la disposición de una sola herramienta de investigación. Entre los criterios para la selección de las personas en el focus group, se ha considerado esencial la participación de estudiantes con resultados diversos en la evaluación final. Para su elección, hemos optado por una selección aleatoria, con la finalidad de obtener resultados objetivos.

Nuestras conclusiones, aunque no se trata de la misma población objeto de estudio, se corresponden con lo reflejado por Miralles, Gómez y Rodríguez (2017) para el profesorado en formación de educación secundaria. Según estos investigadores, casi el 50\% del futuro profesorado de secundaria valora las salidas a lugares de interés histórico por encima de 4 puntos en una escala de 5. Para nuestro caso, aunque se trata de un ámbito particular con una metodología concreta ya implementada, el $85 \%$ está totalmente de acuerdo en que trabajar de forma activa los enclaves históricos y patrimoniales mejora su formación docente y que el $90 \%$ está de acuerdo, y muy de acuedo, en tener presente las visitas e itinerarios para su futura aplicación profesional.

Se puede señalar que la evaluación de nuestro enfoque metodológico presenta resultados muy parecidos a los obtenidos por Bonilla y Lucena (2019), donde se revisa la utilidad de las salidas escolares en un grupo de estudiantes similar, como es el alumnado en formación de educación infantil. En ambos trabajos se observan valoraciones muy positivas respecto al contacto directo con el medio y el cambio de consideración del patrimonio local, lo que también se traduce en un aumento motivacional por conocer el entorno y sus particularidades. La valoración positiva del itinerario didáctico como recurso participativo también ha sido recogida en el ámbito de la formación del profesorado de educación infantil y primaria en la Unviersidad de Sevilla, aunque la secuencia didáctica planteada es diferente a la que aquí proponemos. En el contexto sevillano, el $98 \%$ de las y los participantes ha considerado muy oportuno las salidas fuera del aula en su formación, ya que permiten trabajar la educación patrimonial y los problemas socioambientales relevantes de ámbito local y global (Ferreras-Listán, Moreno-Fernández y Puig, 2019).

Podemos considerar, a través de la información recogida, una asimilación de conceptos más significativa, fruto de la implicación a la que se redirigen las actividades y trabajos del alumnado; una propuesta didáctica que resulta bastante más dinámica que las rutinas del aula, algo que queda constatado tanto en los cuestionarios como en el estudio focal. Los esfuerzos realizados durante el proceso de diseño y desarrollo del itinerario patrimonial se ven recogidos en el proyecto final de la asignatura, donde en la mayor parte de casos se contemplan visitas e itinerarios a realizar fuera del aula, programados para un ámbito concreto de educación infantil.

Consideramos que "desde esta perspectiva didáctica, el itinerario es un instrumento educativo y sensibilizador del patrimonio que permite la interacción directa del individuo con su medio" (Pérez y Morón, 2016, p. 93). No obstante, este recurso didáctico presenta una amplia variedad de 
posibilidades en cuanto a sus objetivos, finalidades, planificación y ejecución; variables que deben ser consideradas en la formación del profesorado. El alumnado recuerda participar de forma pasiva en etapas previas. Sin embargo, concebimos que su colaboración debe ser activa en todos los procesos (Benejam y Martorell, 2003; Vilarrasa, 2003a y 2003b).

Por todo, resulta necesario seguir investigando los procesos y resultados que se obtienen en la formación del profesorado sobre la implementación de metodologías activas; resultados que fundamenten las prácticas de aula y ayuden a avanzar la didáctica de las Ciencias Sociales en general y la educación patrimonial en particular, relacionando la teoría con la práctica (Santisteban y Pagès, 2006).

El contacto con el entorno por medio de itinerarios didácticos, de carácter patrimonial, son una herramienta de gran interés en la formación del futuro profesorado. No obstante, si bien son bastante utilizados a partir de primaria, también tienen un gran potencial en la educación infantil, donde el alumnado debe adquirir las capacidades para desarrollar este recurso adaptado a la realidad de su aula y aprovechar los contextos cercanos. En nuestro caso, entendemos que la metodología utilizada debe ser participativa y colaborativa en todo el proceso, lo que nos permite educar en, desde y para el patrimonio.

Para el desarrollo de la investigación, cabe considerar la predisposición de un grupo control, donde se plantée otra metodología y nos permita evaluar ambos casos. Sin embargo, en este caso puede resultar una falta de ética moral y profesional utilizar al alumnado, en contra de una educación integral y constructivista.

La percepción de los estudiantes avala nuestra propuesta, lo que nos ayuda a continuar implementando esta metodología de trabajo en la educación patrimonial del profesorado que se forma para atender las exigencias de la educación infantil, prestando atención a las necesidades de mejora, con la finalidad de que este pueda desarrollar en su futuro alumnado los conocimientos y valores, históricos y sociales, que están presentes en el territorio.

\section{Referencias}

Alves, L. A. y Pinto, H. (2019). Educación histórica con el patrimonio: desafiando la formación de profesorado. Revista Electrónica Interuniversitaria de Formación del Profesorado, 22(1), 71-81. DOI: 10.6018/reifop.22.1.356381

Arias, L. y Casanova, E. (2018). El uso de los objetos arqueológicos en las aulas de educación infantil: una experiencia en un aula de cuatro años. En A. Egea, L. Arias y J. Santacana (Coords.), Y la arqueología llegó al aula (pp. 121-135). Gijón: Ediciones TREA.

Arias, L., Casanova, E., Egea, A., García, A. y Morales, Mª J. (2016). Aprendiendo a tocar la historia. Las fuentes objetuales como recurso de aprendizaje en educación infantil y primaria. En R. López-Facal (Ed.), Ciencias Sociales, educación y futuro. Investigaciones en didáctica de las ciencias sociales (pp. 136-148). Santiago de Compostela: RED14Universidad de Santiago de Compostela.

Ávila, R.M. y Duarte, O. (2014). Mi patrimonio, mi museo. Una experiencia didáctica en la formación inicial del profesorado de educación infantil. En J. Pagès y A. Santisteban (Eds.). Una mirada al pasado y un proyecto de futuro. Investigación e innovación en didáctica de las ciencias sociales. XXV Simposio de Didáctica de las Ciencias Sociales. Vol. 2 (pp. 371378). Barcelona: AUPDCS/Servei de Publicacions UAB.

Ballesteros, E., Fernández, C., Molina, J. A. y Moreno, P. (Ed.). (2003). El patrimonio y la didáctica de las ciencias sociales. Cuenca: AUPDCS y Universidad de Castilla-La Mancha.

Benejam, P. y Martorell, T. (2003). Los objetivos de las salidas. Iber: Didáctica de las ciencias sociales, geografía e historia, 36, 7-12. 
Bonilla, A.L. y Lucena, C. (2019). Metodología de trabajo en la enseñanza del patrimonio cultural en la facultad de ciencias de la educación (UGR). Universidad, Escuela y Sociedad, UNES, 7 $148-160$. Recuperado

de https://www.revistaunes.com/index.php/revistaunes/article/view/98/88

Calderón, B. (2018). La ciudad educadora. Instrumentos didácticos para el aprendizaje del entorno social y cultural en Educación Infantil mediante proyectos educativos: el ejemplo de Málaga romana. En E. López, C. R. García y M. Sánchez (eds.), Buscando formas de enseñar: investigar para innovar en didáctica de las Ciencias Sociales (pp. 895-906). Valladolid: AUPDCS y Universidad de Valladolid.

Cambil, Ma . E. y Fernández, A. R. (2017). El concepto actual de patrimonio cultural y su valor educativo: fundamentación teórica y aplicación didáctica. En M ${ }^{\mathrm{a}}$. E. Cambil y A. Tudela (coords.), Educación y patrimonio cultural. Fundamentos, contextos y estrategias didácticas (pp. 27-46). Madrid: Pirámide.

Cambil, Ma . E. y Tudela, A. (Coords.) (2017). Educación y patrimonio cultural. Fundamentos, contextos y estrategias didácticas. Madrid: Pirámide.

Candreva, A. y Susacasa, S. (2003). El valor del Patrimonio en el currículo de la formación docente. En E. Ballesteros, C. Fernández, J. A. Molina y P. Moreno (coords.), El Patrimonio y la didáctica de las Ciencias Sociales (pp. 41-50). Cuenca: AUPDCS y Universidad de Castilla-La Mancha.

Casanova, E., Arias, L. y Egea, A. (2018). La metodología por proyectos como oportunidad para la introducción de la historia y el patrimonio en las aulas de educación infantil. Contextos Educativos, 22, 79-95. DOI: 10.18172/con.3185

Casanova, E. y Casanova, J. M. (2014). El patrimonio en educación infantil: una propuesta de acercamiento al patrimonio medieval islámico de la ciudad de Murcia. En P. Miralles, $\mathrm{M}^{\mathrm{a}}$. B. Alfageme y R. Rodríguez (coords.), Investigación e innovación en educación infantil (pp. 249-260). Murcia: Universidad de Murcia.

Castro, L. y López-Facal, R. (2019). Educación patrimonial: necesidades sentidas por el profesorado de infantil, primaria y secundaria. Revista Interuniversitaria de Formación del Profesorado, 94(33.1), 97-114. DOI: 10.47553/rifop.v33i1.72020

Chaparro, A., Martínez, C., Robles, F. J., y Cespedosa, R. (2019). Desarrollar la conciencia histórica a través del patrimonio en el Grado en Educación Primaria. Didáctica de las Ciencias Experimentales y Sociales, 36, 17-32. DOI: 10.7203/DCES.36.12672

Coma, L. (2012). Investigación en didáctica del patrimonio en el marco de las ciudades educadoras. Enseñanza de las ciencias sociales, 11, 53-60.

Cuenca, J. M. y Domínguez, C. (2000). Un planteamiento sociohistórico para educación infantil. El patrimonio como fuente para el trabajo de contenidos temporales. Íber. Didáctica de las Ciencias Sociales, Geografia e Historia, 23, 113-123.

Cuenca, J. Ma . (2003). Análisis de concepciones sobre la enseñanza del patrimonio en la educación obligatoria. Enseñanza de las ciencias sociales, 2, 37-45.

Cuenca, J. Ma . (2014). El papel del patrimonio en los centros educativos: hacia la socialización patrimonial. Tejuelo, 19, 76-96.

Cuenca, J. Ma . y Estepa, J. (2003). El patrimonio en las ciencias sociales. Concepciones transmitidas por los libros de texto de la E.S.O. En E. Ballesteros; C. Fernández; J. A. Molina y P. Moreno (coords.), El patrimonio y la didáctica de las ciencias sociales (pp. 91102). Cuenca: AUPDCS y Universidad de Castilla-La Mancha.

Cuenca, J.M. y Martín, M. J. (2014). Manual para el desarrollo de proyectos educativos de museos. Gijón: Ediciones TREA.

Cuenca, J. Mª, Espeta, J. y Martín, Ma . J. (2017). Patrimonio, educación, identidad y ciudadanía. Profesorado y libros de texto en la enseñanza obligatoria. Revista de Educación, 375, 136159. DOI: $10.4438 / 1988-592 X-R E-2016-375-338$ 
Cuenca, J. Mª, Martín, M. y Schugurensky, D. (2017). Educación para la ciudadanía e identidad en los museos de Estados Unidos. Análisis desde la perspectiva de la educación patrimonial. Estudios Pedagógicos XLIII, 4, 29-48.

Delgado, E. y Alario, Ma . T. (1994). La interacción fuera del aula: itinerarios, salidas y paseos. Tabanque, 9, 155-178.

Domínguez, A. y López, R. (2017). Formación de maestros y educación patrimonial. Estudios Pedagógicos, XLIII, 4, 49-68. DOI: 10.4067/S0718-07052017000400003

Escribano, A. (2015). Utilización del patrimonio para el aprendizaje de la historia en educación infantil. Experiencia educativa "Esta clase es un Museo". Pulso, 38, 179-205.

Estepa, J., Ávila, R. y Ruíz, R. (2007). Concepciones sobre la enseñanza y difusión del patrimonio en las instituciones educativas y los centros de interpretación. Estudio descriptivo. Enseñanza de las ciencias sociales, 6, 75-94.

Estepa, J., Wamba, A. Ma y Jiménez, R. (2005). Fundamentos para una enseñanza y difusión del patrimonio desde una perspectiva integradora de las ciencias sociales y experimentales. Investigación en la escuela, 56, 19-26.

Feliu, M., Jiménez, L. y Arqué, Ma . T. (2014). El trabajo cooperativo en el grado de educación infantil. Elaboración de recursos didácticos para el descubrimiento del entorno. Enseñanza de las ciencias sociales, 13, 153-160.

Fernández, M. (2003). Los museos espacios de cultura, espacios de aprendizaje. Iber, Didáctica de las Ciencias Sociales, Geografia e Historia, 36, 55-61.

Fontal, O. (2003). La educación patrimonial. Teoría y práctica en el aula, el museo e Internet. Gijón: Trea.

Fontal, O. (2017). El patrimonio cultural del entorno próximo: un diseño de sensibilización para secundaria. Enseñanza de las ciencias sociales, 6, 31-47.

Fontal, O. e Ibáñez, A. (2015). Estrategias e instrumentos para la educación patrimonial en España. Educatio Siglo XXI, 33(1), 15-32. DOI: 10.6018/j/222481

Fontal, O., Ibáñez, A., Martínez, M. y Rivero, P. (2017). El patrimonio como contenido en la etapa de primaria. Revista electrónica interuniversitaria de Formación del Profesorado, 20(2), 79-95. DOI: 10.6018/reifop.20.1.286321

Franco, C. y Huerta, R. (2011). La creación de una mirada urbana. La ciudad de Santiago de Compostela interpretada por el alumnado de magisterio. Educatio Siglo XXI, 29(2), 229246.

Fuertes, C. (2016). Emoción, oralidad e itinerarios didácticos: un estudio de caso en el grado en maestro de educación infantil. Revista de Didácticas Especificas, 15, 51-69.

Gómez, C. (2014). Nuevos entornos en la formación superior: una propuesta de educación patrimonial en el museo. Profesorado, revista de currículum y formación del profesorado, $18(3), 293-317$.

González-Monfort, N. (2008). Una investigación cualitativa y etnográfica sobre el valor educativo y el uso didáctico del patrimonio cultural. Enseñanza de las ciencias sociales, 7, 23-36.

González-Monfort, N. (2011). La presencia del patrimonio cultural en los currícula de educación infantil, primaria y secundaria obligatoria en España. Patrimonio Cultural de España, 5, 5974.

González-Monfort, N. (2019). La educación patrimonial, una cuestión de futuro. Reflexiones sobre el valor del patrimonio para seguir avanzando hacia una ciudadanía crítica. El futuro del pasado, 10, 124-144. DOI: 10.14516/fdp.2019.010.001.004.

Hernández, A. Ma . y Guillén, R. (2017). La educación patrimonial en los manuales escolares de Educación Primaria: un recorrido desde LOGSE hasta LOMCE. Didáctica de las Ciencias Experimentales y Sociales, 32, 25-.49. DOI: 10.7203/DCES.32.9205

Hernández, F. X. (2003). El patrimonio como recurso en la enseñanza de las ciencias sociales. En E. Ballesteros; C. Fernández; J. A. Molina y P. Moreno (coords.), El patrimonio y la 
didáctica de las ciencias sociales (pp. 455-466). Cuenca: AUPDCS y Universidad de Castilla-La Mancha.

Hernández, L. y Pagès, J. (2016). ¿Cómo enseñar historia y ciencias sociales en la educación preescolar? Revista Mexicana de Investigación Educativa, 21(68), 119-140.

Ibáñez, A., Fontal, A. y Cuenca, J. Ma . (2015). Actualidad y tendencias en Educación Patrimonial. Educatio Siglo XXI, 33, 11-14. Recuperado de https://revistas.um.es/educatio/article/view/222471

Inhelder, B., Bovet, M., Sinclair, H. y Smock, C.D. (1966). On cognitive development. American psychologists, 21(2), 160-164.

Insa, Y. (2002). Itinerarios urbanos, recursos y materiales didácticos para explicar la ciudad. Íber Didáctica de las ciencias sociales: Geografía e Historia, 32, 89-95.

Lleida, M. (2010). El patrimonio arquitectónico, una fuente para la enseñanza de la historia y las ciencias sociales. Enseñanza de las ciencias sociales, 9, 41-50.

López, F. y Segura, J. (2013). Los itinerarios didácticos: un recurso interdisciplinar y vertebrador del currículum. Espiral, Cuadernos del Profesorado. Revista Digital del Centro de Profesorado Cuevas-Olula, 6(12). Recuperado de http://www.cepcuevasolula.es/espiral

López, J. A. (2020). Concepciones sobre el patrimonio en la formación del profesorado de educación infantil: un recurso para conocer el entorno. En A. Ruíz, S. Medina y L. Pérez (coords.), Educación y divulgación del patrimonio arqueológico. La socialización del pasado como reto para el futuro (pp. 1-19). Granada: Ed. Comares.

Luna, U., Vicent, N., Reyes, W., y Quiñonez, S. H. (2019). Patrimonio, currículum y formación del profesorado de Educación Primaria en México. Revista Electrónica Interuniversitaria de Formación del Profesorado, 22(1), 83-102. DOI: 10.6018/reifop.22.1.358761

Mateo, M. R. (2019). Las salidas de campo en el medio rural. Análisis de las investigaciones presentadas a los congresos del grupo de trabajo de didáctica de la geografía de la AGE y de la APG (1988-2016). En X.C. Macía, F. X. Armas y F. Rodríguez (coords), La reconfiguración del medio rural en la sociedad de la información (pp. 1279-1292). Santiago de Compostela: Andavira

Medina-Quintana, S. y López-Fernández, J. A. (2018). El patrimonio en la formación del profesorado de Educación Infantil: una propuesta activa a través del casco histórico de Córdoba. En E. López-Torres, C. R. García-Ruíz y M. Sánchez-Agustí (coords.), Buscando formas de enseñar: investigar para innovar en Didáctica de las Ciencias Sociales (pp. 263274). Valladolid: Universidad de Valladolid/AUPDCS.

Medina-Quintana, S., Arrebola, J. C., Mora, M. y López, J. A. (2016). Propuesta de itinerario interdisciplinar en la formación del profesorado de Educación Primaria en el ámbito de las ciencias sociales y experimentales. Didáctica de las Ciencias Experimentales y Sociales, 31 , 79-97. DOI: $10.7203 /$ dces.31.8058

Ministerio de Educación, Cultura y Deporte (2015). Plan Nacional de Educación y Patrimonio. Secretaría General Técnica. Recuperado de http://www.culturaydeporte.gob.es/planesnacionales/planes-nacionales/educacion-y-patrimonio.html

Miralles, P. y Rivero, P. (2012). Propuestas de innovación para la enseñanza de la historia en Educación Infantil. Revista Electrónica Interuniversitaria de Formación del Profesorado, 15(1), 81-90.

Miralles, P., Gómez, C. y Rodríguez, R. (2017). Patrimonio, competencias históricas y metodologías activas de aprendizaje. Un análisis de las opiniones de los docentes en formación en España e Inglaterra. Estudios Pedagógicos XLIII, 4, 161-184. DOI: 10.4067/S0718-07052017000400009

Miralles, P., Molina, S. y Ortuño, J. (2011). La Investigación en didáctica de las ciencias sociales. Educatio Siglo XXI, 29, 149-174. 
Molina, S. y Muñoz, R. (2016). La opinión del profesorado de Educación Secundaria sobre el papel del patrimonio en la enseñanza de las Ciencias Sociales: un estudio de caso. Revista Complutense de Educación, 27(2), 863-880. DOI: 10.5209/rev_RCED.2016.v27.n2.48411

Molina, S. y Ortuño, J. (2017). Concepciones del profesorado iberoamericano de Secundaria sobre la contribución del patrimonio local al desarrollo del pensamiento histórico. Estudios Pedagógicos, XLIII (4), 185-202. DOI: 10.4067/S0718-07052017000400010

Morón, H. y Morón, M. C. (2017). La evolución del Concepto de Patrimonio: Oportunidades para la enseñanza de las ciencias. Didáctica de las ciencias experimentales y sociales, 33, 83-98. DOI: $10.7203 /$ dces.33.10814

Olave, D. (2005). El itinerario didáctico: una propuesta metodológica para el análisis geo-histórico local. Geoenseñanza, 10, 197-208.

Pagès, J. (2000). La formación inicial del profesorado para la enseñanza del patrimonio histórico y de la historia. III Seminari Arqueologia i Ensenyament, Treballs d'Arqueologia, 6, 205-217.

Pérez, M. y Morón, H. (2016). El itinerario didáctico como herramienta para la activación patrimonial: una experiencia docente desde el parque María Luisa. Campo Abierto, 35(2), 83-94.

Piaget, J. (1954). The child's construction of reality. Nueva York: Basic Books.

Pinto, H. y Molina, S. (2015). La educación patrimonial en los currículos de ciencias sociales de España y Portugal. Educatio Siglo XXI, 33(1), 103-128. DOI: 10.6018/j/222521

Piotrowska, I., Cichoń, M., Abramowicz, D. y Sypniewski, J. (2019). Challenges in geography education - a review of research problems. Quaestiones Geographicae 38(1), 71-84. DOI: 10.2478/quageo-2019-0009

Prats, J. (2001). Valorar el patrimonio histórico desde la educación: factores para una mejor utilización de los bienes patrimoniales. ICE de la Universidad de Zaragoza.

Prats, J. (2003). Líneas de investigación en didáctica de las ciencias sociales. História Ensino Revista do Laboratório de Ensino de Históriória/UEL, 9, 1-25.

Santacana, J. (2006). Bases para una museografía didáctica en los museos de arte. Enseñanza de las ciencias sociales, $5,125-133$.

Santacana, J. y Martínez, T. (2013). Patrimonio, identidad y educación: una reflexión teórica desde la historia. Educatio Siglo XXI, 31(1), 47-60.

Santacana, J., Martínez, T., Llonch, N. y López, V. (2016). ¿Qué opinan los adolescentes sobre los museos y la didáctica? Didáctica de las ciencias experimentales y sociales, 31, 23-38. DOI: $10.7203 /$ dces.31.8795

Santisteban, A. (2000). Aprender a enseñar el tiempo histórico: esquemas de conocimiento y perspectiva práctica en el alumnado de formación inicial. En J. Pagès, J. Estepa y G. Travé (eds.), Modelos, contenidos y experiencias en la formación del profesorado de Ciencias Sociales (pp. 355-369). Huelva: Universidad de Huelva-AUPDCS.

Santisteban, A. y Pagès, J. (2006). La enseñanza de la historia en Educación Primaria. En Educación Primaria: orientaciones y recursos (6-12 años) (pp- 129-183). Barcelona: CISSPraxis.

Santolaria, A. (2014). La ciudad como recurso didáctico. Edetaina, 45, 235-244.

Tonda, E. Ma . (2001). La didáctica de las Ciencias Sociales en la formación del profesorado de educación infantil. Alicante: Publicaciones de la Universidad de Alicante.

Trepat, C. A., y Comes, P. (2000). El tiempo y el espacio en la didáctica de las ciencias sociales. Barcelona: Graó, $3^{\mathrm{a}}$ ed.

Vilarrasa, A. (2003a). Salir del aula. Íber. Didáctica de las ciencias sociales, geografía e historia, $36,5-6$.

Vilarrasa, A. (2003b). Salir del aula. Reapropiarse del contexto. Íber. Didáctica de las ciencias sociales, geografía e historia, 36, 13-25. 


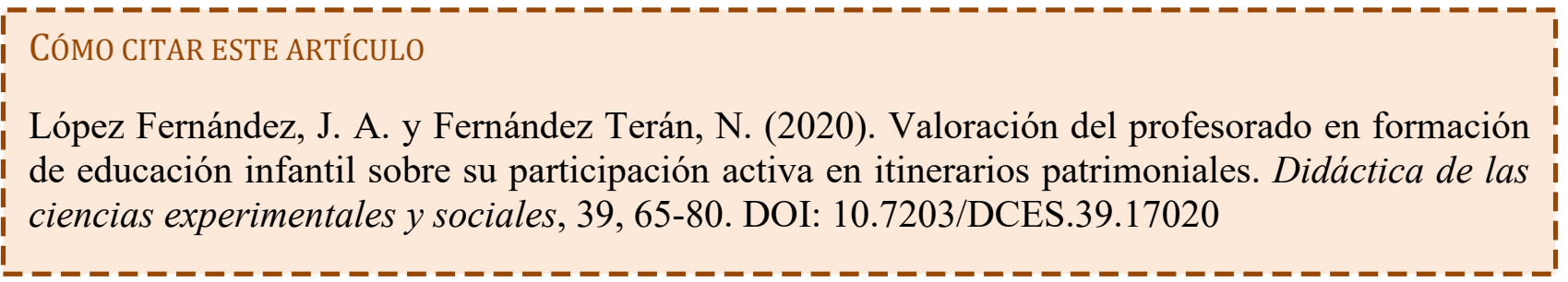

